\title{
Validation of the CALUX bioassay as a screening and semi-quantitative method for PCDD/F levels in cow's milk
}

\author{
I-Cheng Chou ${ }^{a, b}$, Wen-Jhy Lee ${ }^{a, b, *}$, Lin-Chi Wang ${ }^{c}$, Guo-Ping Chang-Chien ${ }^{c}$, \\ Wei-Shan Lee ${ }^{\mathrm{c}}$, Hsinyu Lee ${ }^{\mathrm{d}}$ \\ ${ }^{a}$ Department of Environmental Engineering, National Cheng Kung University, Tainan 70101, Taiwan \\ b Sustainable Environment Research Center, National Cheng Kung University, Tainan 70101, Taiwan \\ ${ }^{c}$ Department of Chemical and Materials Engineering, Cheng Shiu University, Kaohsiung 833, Taiwan \\ ${ }^{\mathrm{d}}$ Department of Life Science, National Taiwan University, Taipei 106, Taiwan
}

Received 23 April 2007; received in revised form 13 November 2007; accepted 13 November 2007

Available online 17 November 2007

\begin{abstract}
The objectives of this study were first to set up and validate the quality criteria of the Xenobiotic Detection Systems—chemical activated luciferase gene expression (CALUX) bioassay for the analysis of cow's milk samples spiked with polychlorinated dibenzo- $p$-dioxins and polychlorinated dibenzofurans (PCDD/Fs). The application of this bioassay was then tested by analyzing 28 commercially available pasteurized milk samples and comparing the data with the reference method for PCDD/F analysis. The CALUX criteria from the U.S. EPA and the European Union (EU) were calculated from 16 replaced spiked milk (SM) samples and 8 performance evaluation (PE) samples to validate the CALUX bioassay system. The CALUX bioassay criteria included control chart for quality control (QC) standards, recovery efficiency, and data comparability. The control chart for QC standards were both within the $\mu \pm 2 \sigma$ range. The recovery efficiencies ranged from $60.4 \%$ to $106 \%$ with an average of $79.9 \%$ (relative standard deviation (RSD): 20.7\%). The mean of data comparability (i.e., relative percent difference, RPD) between CALUX bioassay and high-resolution gas chromatography and high-resolution mass spectrometry (HRGC/HRMS) was 19.0\% for SM samples. For the PE samples, Pearson's correlation coefficient between CALUX and the HRGC/HRMS method was 0.953 . The high correlation shows that the CALUX system is suitable as a screening method and a semi-quantitative method to analyze the PCDD/F concentration in milk samples. Next, the validated CALUX bioassay was applied to measure 28 commercially available pasteurized milk samples. These milk samples were also analyzed with the HRGC/HRMS method to compare the analysis data from two different methods. There is no false negative sample when applying the bioassay to pasteurized milk and PE samples as a screening method and a semi-quantitative method. The present study indicates that CALUX is a powerful bioassay method for screening a large number of milk samples.
\end{abstract}

(C) 2007 Elsevier B.V. All rights reserved.

Keywords: PCDDs; PCDFs; Cow's milk; CALUX; Bioassays

\section{Introduction}

Humans are exposed to various persistent organic pollutants (POPs) in daily life. These POPs can cause serious diseases and adverse bioaccumulation effects in the environment, with polychlorinated dibenzo- $p$-dioxins (PCDDs) and polychlorinated dibenzofurans (PCDFs) of particular concern.

\footnotetext{
* Corresponding author at: Department of Environmental Engineering, National Cheng Kung University, No. 1 University Road, Tainan City 70101, Taiwan. Tel.: +886 $62757575 x 65831$; fax: +886 62752790 .

E-mail address: wjlee@mail.ncku.edu.tw (W.-J. Lee).
}

2,3,7,8-Tetrachlorodibenzo-p-dioxin (2,3,7,8-TCDD) has been categorized as a group 1 agent (i.e., carcinogenic to humans) by the International Agency for Research on Cancer (IARC). 2,3,7,8-TCDD has been shown to be a risk factor for soft-tissue sarcoma, non-Hodgkin lymphoma, malignant neoplasm [1], and cancer [2], although it is not a direct genotoxin [1]. One of the 2,3,7,8-TCDD genotoxic hypotheses is the aryl hydrocarbon receptor (AhR)-mediated alteration in the expression of networks of genes involved in cell growth and differentiation [1]. The chemical activated luciferase gene expression (CALUX) bioassay was established (from Xenobiotic Detection Systems) for detecting the AhR-mediated luciferase expression induced by those dioxin-like chemicals. 
A food survey in 2004 from the Department of Health Taiwan (TDOH) in which the contaminated pasteurized milk showed a PCDD/F level higher than $3.0 \mathrm{pg}-\mathrm{WHO}-\mathrm{TEQ} / \mathrm{g}$ fat, exceeded the limit imposed by the European Union (EU). In the same year, the TDOH released a draft concentration limit for PCDD/Fs in pasteurized milk ( $3 \mathrm{pg}-\mathrm{WHO}-\mathrm{TEQ} / \mathrm{g}$ fat) in response to concerns from the public. TDOH further confirmed the regulation in 2006. This affair showed that the public is highly concerned with the issue of PCDD/Fs in commercial pasteurized cow's milk.

The traditional high-resolution gas chromatography and high-resolution mass spectrometry (HRGC/HRMS) analysis is a chemical method commonly used to measure the PCDD/F concentration of pasteurized milk. The advantages of the HRGC/HRMS analysis are its high accuracy and good reliability for the separation of individual PCDD/F congeners. The HRGC/HRMS method requires 4-6 weeks to obtain the $\mathrm{PCDD} / \mathrm{F}$ concentration in a pasteurized milk sample. However, the recommended storage time for pasteurized fresh milk, at $4-7{ }^{\circ} \mathrm{C}$ is approximately $7-10$ days if the container is not opened [3].

The CALUX bioassay used in this study is an in vitro luciferase assay, which can be used to measure the PCDD/F concentration of pasteurized milk within 3-7 days. The major advantage of the CALUX bioassay is that it directly determines the total sum of dioxin toxic equivalency (TEQ) in the test samples. In fact, similar CALUX bioassay have been applied to measure the content of $\mathrm{PCDD} / \mathrm{Fs}$ in various matrices, including marine life (eels [4,5], mussels [6], and fish [7]), foodstuff (beef [8], cod liver [8], and milk [5]), feed [8,9], human tissue (blood plasma [10,11] and human milk [12]), and environmental media (soil and sediment [13]). However, few studies have conducted the measurement of PCDD/F in cow's milk using the CALUX. Moreover, different matrices could contain some materials (e.g., metals) that could affect the reading of CALUX bioassay $[14,15]$.

The objective of this study was to set up and validate the quality criteria of the CALUX bioassay for analyzing dioxin levels in the PCDD/F-spiked cow's milk samples. The application of this bioassay was then tested by analyzing $28 \mathrm{com}$ mercially available pasteurized milk samples and comparing the results with the reference method for PCDD/F analysis.

\section{Materials and methods}

\subsection{Chemicals}

The EPA-1613 stock (17 PCDD/F congener standards) and 2,3,7,8-TCDD were purchased from Wellington Laboratories (Canada). Acetone, sulfuric acid, and silica gel were purchased from Merck (Germany). Celite and anhydrous sodium sulfate were purchased from Sigma-Aldrich (USA). $1 \%$ $\mathrm{XCARB} / \mathrm{Celite}$ was from XDS (USA). Toluene and $n$-hexane were purchased from TEDIA (USA). The Luciferase Assay System (luciferase reagent) was purchased from Promega Corp. (USA).

\subsection{Sample collection and preparation}

A total of 28 full-fat pasteurized milk samples produced by 8 different companies were collected from two chain stores and two supermarkets in Southern Taiwan. The fat content of each sample ranged from $3.3 \%$ to $4.1 \%$, with an average of $3.7 \%(\mathrm{w} / \mathrm{w})$. Each sample was labeled and stored at $4{ }^{\circ} \mathrm{C}$ until extraction.

One of the milk samples was spiked with a PCDD/F standard solution up to $3 \mathrm{pg}-\mathrm{WHO}-\mathrm{TEQ} / \mathrm{g}$ fat as a constant concentration spiked milk (SM) sample. To determine the recovery efficiency of the pretreatment and the stability of the CALUX bioassay, the SM sample was separated into 16 samples (SM1-SM16) for the bioassay. The performance evaluation (PE) samples (PE0, PE1, PE2, PE3, and PE4) were produced by taking $100 \mathrm{~mL}$ of each pasteurized milk sample, and spiked with the PCDD/F standard solution to levels of $0,1,2,3$, and $4 \mathrm{pg}$-WHO-TEQ/g fat, respectively. Each PE sample was divided into 2 aliquots for the CALUX analysis.

\subsection{CALUX bioassay}

The cell line used in the bioassay was a modified cell line [10], H1L6.1c2, which was obtained from Hiyoshi Corporation (Japan) and is the same as that from Xenobiotic Detection Systems (XDS, USA) [16]. CALUX bioassay was performed as previously described [17], except for the extraction method and clean-up procedure.

The volume of the milk samples was $60 \mathrm{~mL}$. Acetone was used to extract milk fat from individual pasteurized milk samples with liquid-liquid extraction. The water and impurities in samples were removed by passing through an extraction column, which contained celite and anhydrous sodium sulfate. Nitrogen blowing was used to completely remove the solvent and water from the extract. The fat was passed through an acid silica gel column and an XCARB column to further extract the milk fat. The acid silica gel column consisted mainly of $45 \%$ (w/w) sulfuric acid silica gel and anhydrous sodium sulfate. The XCARB column consisted mainly of $1 \% \mathrm{XCARB} / \mathrm{Celite}$ and anhydrous sodium sulfate. The PCDD/F samples from the XCARB column were collected, dissolved in toluene, and concentrated to dryness using the vacuum centrifuge concentrator (JOUAN RC10.10, France). The pretreated PCDD/F samples were dissolved in $4 \mathrm{~mL} n$-hexane and then were stored at $4{ }^{\circ} \mathrm{C}$ until analysis.

Luciferase activity was measured by using a Wallac VICTOR $^{3} 1420$ Multilabel Counter coupled with a dispenser (PerkinElmer Inc., USA) to automatically inject the luciferase reagent. The 2,3,7,8-TCDD calibration curve was generated from the CALUX readings resulted from a serial dilution of 2,3,7,8-TCDD standards $(250,125,62.5,31.3,15.6$, $7.81,3.91,1.95,0.977,0.488,0.244 \mathrm{pg}-\mathrm{TCDD} / \mathrm{mL}$ medium, STD1-STD11) by fitting into a Hill's 4 parameters sigmoid curve. The CALUX results of quality control (QC) standard ( $0.250 \mathrm{ng} \mathrm{I}-\mathrm{TEQ} / \mathrm{mL}$ DMSO diluted from the 1613 stock) and samples were extrapolated into 2,3,7,8-TCDD TEQ values by using the calibration curve. 


\subsection{HRGC/HRMS analysis}

The HRGC/HRMS method that was chosen as the reference method to validate the data of the CALUX bioassay was adopted from the Taiwan Environmental Protection Administration NIEA M801.11B [18] and U.S. EPA Method-1613B [19].

The pretreatments and analyses of milk samples were performed in the Super Micro Mass Research and Technology Center, Cheng Shiu University, Taiwan. The PCDD/F analysis laboratory in Cheng Shiu University has been certified by Taiwan's EPA. The reference method used to determine the PCDD/F concentration in milk samples has been certified by Taiwan Accreditation Foundation (TAF). The clean-up procedure was performed with a Power-Prep ${ }^{\mathrm{TM}}$ automated clean-up system equipped with a multi-layer silica column, an alumina column, and a carbon column (Fluid Management Systems, USA).

The HRGC (Agilent 6890 Series gas, USA) was equipped with a DB-5 MS fused silica capillary column $(60 \mathrm{~m} \times 0.25 \mathrm{~mm}$, film thickness $=0.25 \mu \mathrm{m})(\mathrm{J} \& \mathrm{~W}$ Scientific, USA) with splitless injection. Helium was used as a carrier gas. The HRMS (Micromass Autospec Ultima, Manchester, UK) was equipped with a positive electron impact (EI+) source. The analyzer mode of the selected ion monitoring (SIM) was used with a resolving power of 10,000. The electron energy and source temperature were specified at $35 \mathrm{eV}$ and $250^{\circ} \mathrm{C}$, respectively. The QA/QC criteria followed U.S. EPA Method-1613B [19].

\section{Results and discussion}

\subsection{Quality control criteria for the CALUX bioassay}

Because the internal standard of PCDD/F (i.e., ${ }^{13} \mathrm{C}$-labeled PCDD/F congeners) could be harmful to the H1L1.6 cells, quality control criteria were used to make sure that the CALUX bioassay system was reliable for quantification of the PCDD/F concentration in the pasteurized milk samples. For the reproducibility and consistency of detecting CALUX-TEQs in this bioassay's system, QC standard and PE samples were used to make sure quality control of the samples [20].

The validation of extremely sensitive responses for PCDD/F measurement in this commercial CALUX bioassay were done as previously described [17] by repeatedly analyzing the calibration standards $(n=5)$. The lowest concentration of the coefficient of variation $(\mathrm{CV})<30 \%$ was defined as the limit of quantitation (LOQ). The lowest concentration of $\mathrm{CV}<20 \%$ was defined as the limit of detection (LOD). As shown in Table 1, the LOD and the LOQ values in our CALUX bioassay were 0.488 and $0.977 \mathrm{pg} / \mathrm{mL}$ medium, respectively. The values of LOD and LOQ could also calculated to be 0.168 and $0.335 \mathrm{pg}$ $\mathrm{TEQ} / \mathrm{g}$ fat, respectively, based on the assumption with an average $3.7 \%$ of milk fat and $60 \mathrm{~mL}$ cow's milk. Quantification of the sigmoid calibration curve ranged from 0.977 to $62.5 \mathrm{pg} / \mathrm{mL}$ medium.

The QC standard, i.e., QC chart- and calibration-standard solution (STD6), was used to monitor the quality of calibration

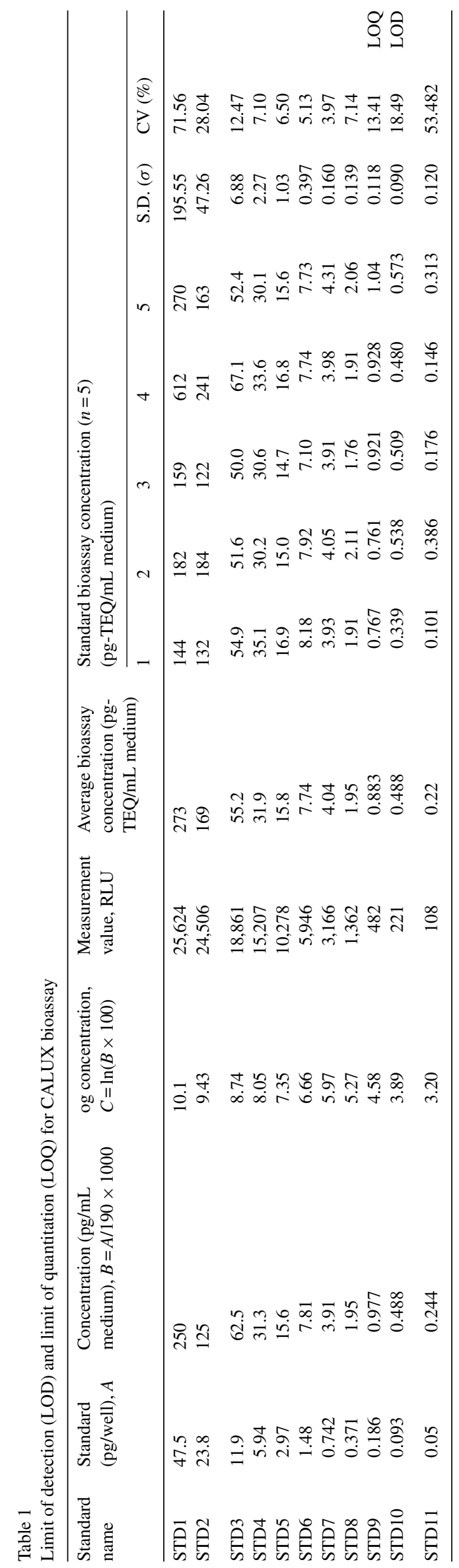



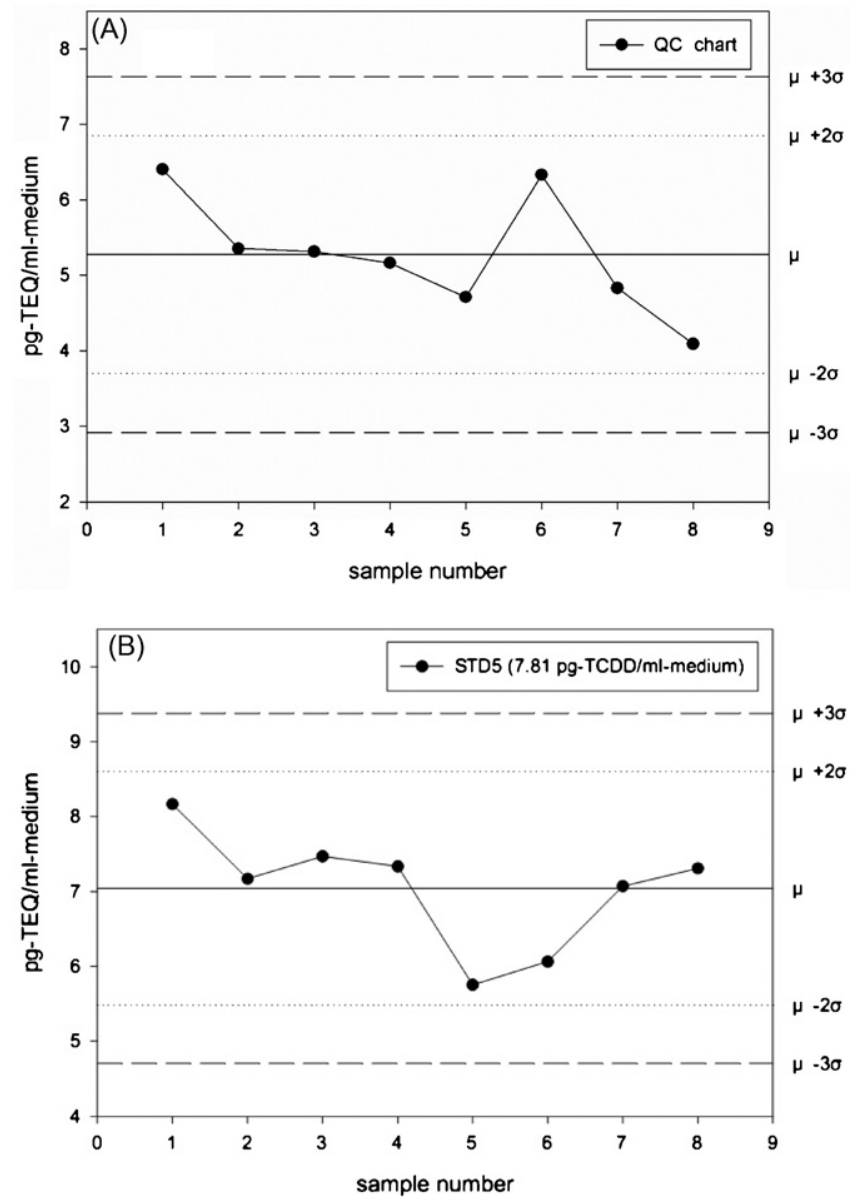

Fig. 1. Control chart for the (A) QC chart and the (B) STD6 standard.

curve and the sensitivity variation of CALUX bioassay in each plate. The Shewhart control charts are shown in Fig. 1(A) and (B). Both control charts followed the decision rules for detecting non-random patterns on control charts that were suggested in the Western Electric Handbook [21]. According to the decision rules, the control limits were $\mu \pm 3 \sigma$ and the warning limits were $\mu \pm 2 \sigma$. The CALUX bioassay and the standard solutions were re-checked when the QC standard was not within the warning limits. The measured CALUX-TEQ is two times higher than the I-TEQ of the QC chart standard solution (see Fig. 1(A)). Because the CALUX relative potencies (REP) values are actual toxicity equivalency factor (TEF) values for the congeners in the CALUX bioassay they represent the actual potency of the specific congener to activate the AhR pathway [23].

\subsection{Quality control criteria for milk analysis}

Before analyzing the pasteurized milk samples, $16 \mathrm{SM}$ samples were analyzed by using CALUX bioassay to examine their results. Fig. 2 shows the control chart of the 16 SM samples. Similar to QC standards, all the data were within the warning limits. As shown in Table 2, the CALUX bioassay data range from 3.99 to 8.62 , with an average of $6.49 \mathrm{pg}-\mathrm{CALUX}-\mathrm{TEQ} / \mathrm{g}$ fat. European Union suggested that the relative standard deviation (RSD)

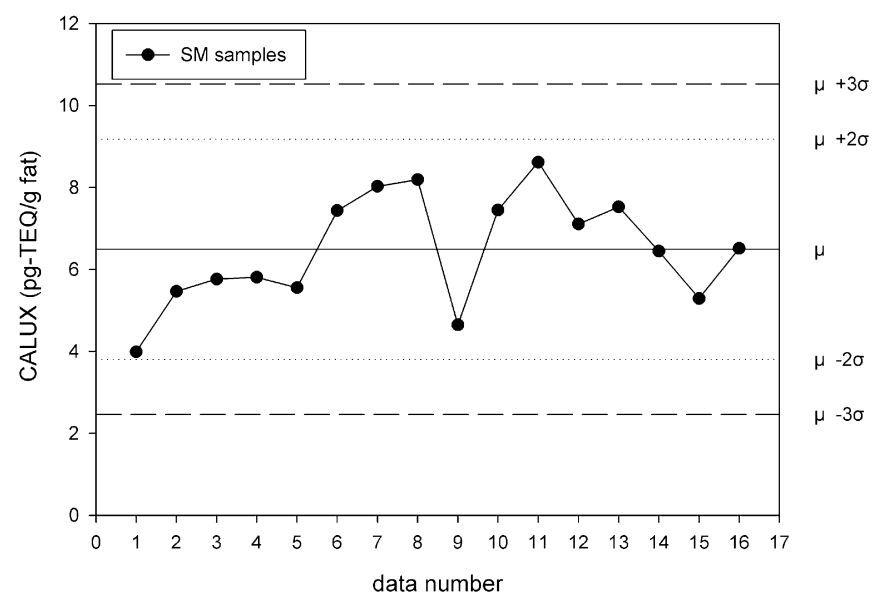

Fig. 2. Control chart of 16 constant concentration spiked milk (SM) samples $(\mathrm{RSD}=20.7 \%)$.

from the precision test of CALUX bioassay needed to be smaller than $30 \%$ [25], and when the value is less than $20 \%$, the U.S. EPA defined that the precision test of the CALUX bioassay is high [13]. The RSD of SM samples was $20.7 \%$, indicating that the precision of CALUX bioassay was acceptable. The control chart also conformed to the decision rules of Western Electric [21].

The PCDD/F recovery efficiency was defined as the ratio of CALUX-TEQs of SM sample to the CALUX-TEQs of standard solution without pretreatment process. The $\mathrm{PCDD} / \mathrm{F}$ recovery efficiencies of SM samples for CALUX ranged from $60.4 \%$ to $106 \%$ with an average of $79.9 \%$, and were within the criteria range of the internal standards for HRGC/HRMS (from $40 \%$ to $130 \%$ ), indicating that $\mathrm{PCDD} / \mathrm{F}$ recovery efficiencies of the CALUX bioassay for pasteurized milk samples were acceptable.

PE samples were spiked at different levels in the HRGC/ HRMS analysis and CALUX bioassay in Fig. 3. Because CALUX-TEF were mostly based on AhR binding affinity, some

Table 2

The CALUX bioassay values of milk SM samples spiked up to $3 \mathrm{pg}$-WHOTEQ/g fat

\begin{tabular}{lll}
\hline Sample number & $\begin{array}{l}\text { XDS-CALUX } \\
(\mathrm{pg}-\mathrm{TEQ} / \mathrm{g} \text { fat })\end{array}$ & $\begin{array}{l}\text { XDS-CALUX } \times \mathrm{CF}^{\mathrm{a}} \\
(\mathrm{pg}-\mathrm{TEQ} / \mathrm{g} \text { fat })\end{array}$ \\
\hline SM1 & 3.99 & 2.07 \\
SM2 & 5.47 & 2.83 \\
SM3 & 5.76 & 2.98 \\
SM4 & 5.81 & 3.01 \\
SM5 & 5.56 & 2.88 \\
SM6 & 7.44 & 3.85 \\
SM7 & 8.03 & 4.16 \\
SM8 & 8.19 & 4.24 \\
SM9 & 4.64 & 2.40 \\
SM10 & 7.45 & 3.86 \\
SM11 & 8.62 & 4.47 \\
SM12 & 7.11 & 3.68 \\
SM13 & 7.53 & 3.90 \\
SM14 & 6.45 & 3.34 \\
SM15 & 5.29 & 2.74 \\
SM16 & 6.52 & 3.38 \\
\hline
\end{tabular}

\footnotetext{
${ }^{\mathrm{a}} \mathrm{CF}=0.518$.
} 


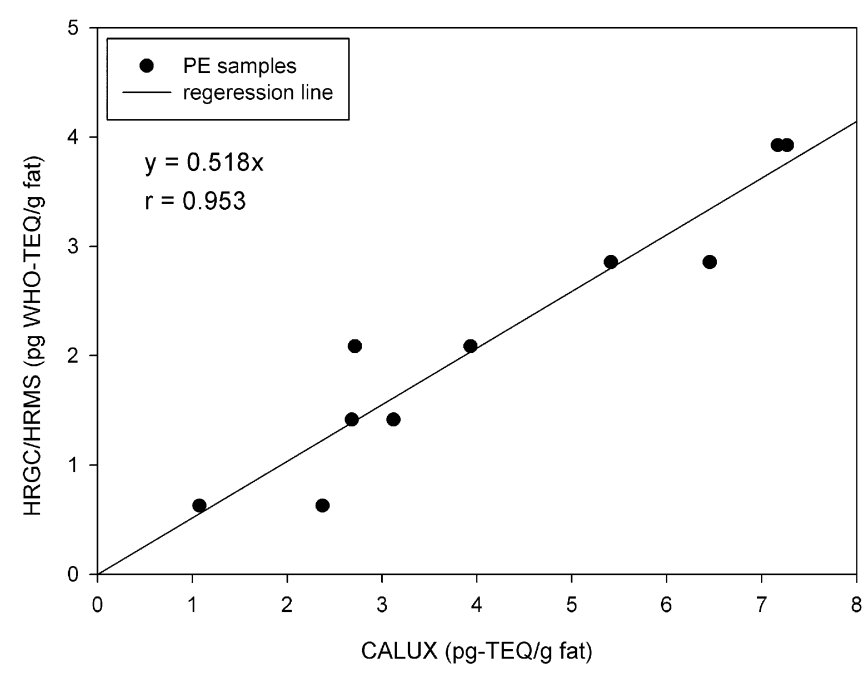

Fig. 3. CALUX results vs. HRGC/HRMS results for spiked performance evaluation (PE) samples.

other compounds like polybrominated dibenzodioxins (PBDDs) and polybrominated dibenzofurans (PBDFs) being AhR ligands may contribute to outcomes of CALUX bioassay. Additionally, non-AhR ligands may also reduce [14] or promote [15] the luciferase light in a similar CALUX bioassay system. Thus, the variation of dioxin-like compounds in the clean-up systems should be possibly eluted before CALUX bioassay tests [22]. Although the values measured by the CALUX and HRGC/HRMS are not the same, however, the result of Pearson's correlation of PE samples revealed that these two methods were highly correlated $(r=0.953, p<0.01)$ (see Fig. 3). Moreover, a conversion factor $(\mathrm{CF})$ could be used to predict dioxin-TEQ levels by HRGC/HRMS method from the values of CALUX-TEQ determined by CALUX bioassay according to a good correlation coefficient of 0.953 .

The value of CF between HRGC/HRMS and CALUX in 10 PE samples (shown in Fig. 3) was $0.518(p<0.01)$, which is higher than that in 28 pasteurized samples $(0.409, p<0.01)$. Compared to the matrices of flue gas $(\mathrm{CF}=0.253)$, fly ash and bottom ash $(\mathrm{CF}=0.345)$ [17], fish-oil $(\mathrm{CF}=0.700)$ [23], the $\mathrm{CFs}$ of biological matrices are higher than those of environmental matrices.

If we recognize CALUX bioassay as one of the semiquantitative methods, the CALUX-TEQ levels of SM samples are transformed to I-TEQ levels based on the assumption that we multiply CALUX-TEQ levels by a CF (CALUX $\times \mathrm{CF})$ as shown in Table 2 with a mean \pm S.D. of $3.36 \pm 0.70 \mathrm{pg}-\mathrm{TEQ} / \mathrm{g}$ fat, respectively.

The definition of accuracy ( $R$-value) [13] is the mean concentration value calculated from the CALUX replicate measurements divided by the HRGC/HRMS value, and the ideal value is $100 \%$. The relative percent difference (RPD) values ranged from $-25 \%$ to $+25 \%$ indicating good agreement between the values of CALUX $\times \mathrm{CF}$ and HRGC/HRMS measurements [13]. For SM samples, the $R$-value was $112 \%$ and the RPD values ranged from $0.32 \%$ to $39.3 \%$ (mean $19.0 \%$ ) when the $\mathrm{CF}$ was 0.518 .

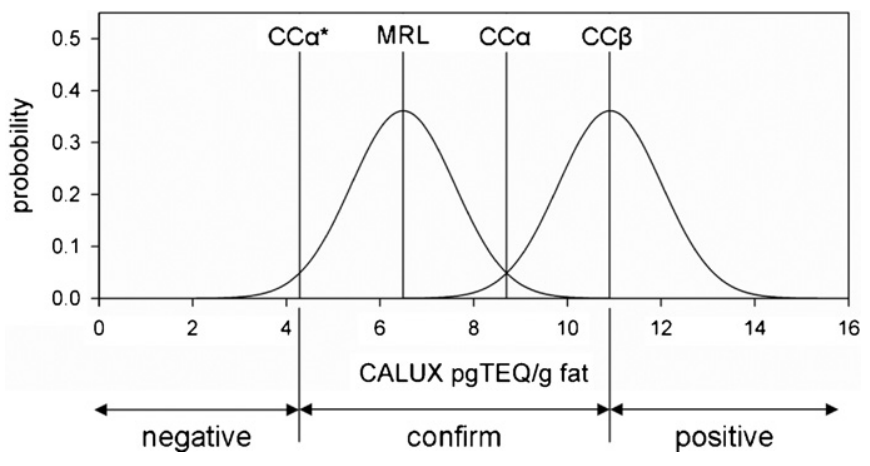

Fig. 4. Graphical representation of $\mathrm{CC} \alpha, \mathrm{CC} \beta$, and $\mathrm{CC} \alpha *$ for analyzing pasteurized milk samples using the CALUX bioassay.

As shown in Fig. 4, the values of 16 SM samples determined by the CALUX bioassay have a normal distribution with a confidence level of $95 \%$. Considering that the probability of type I and II error were the same $(\alpha=\beta=5 \%$ ) [24], the confirmation range was between $4.28 \mathrm{pg}-\mathrm{TEQ} / \mathrm{g}$ fat $\left(\mathrm{CC} \alpha^{*}\right)$ and $10.9 \mathrm{pg}-\mathrm{TEQ} / \mathrm{g}$ fat $(\mathrm{CC} \beta)$. When CALUX-TEQ is higher than $\mathrm{CC} \alpha^{*}$ and lower than CC $\beta$, the samples should be confirmed by HRGC/HRMS method. A value lower than $\mathrm{CC} \alpha^{*}$ is regarded as a negative result, while that higher than $\mathrm{CC} \beta$ is regarded as a positive one. Among the 16 SM samples, only one (SM1) was a negative result, indicating that the CALUX bioassay is ideal for screening milk samples.

\subsection{Analysis of pasteurized milk samples}

As described in Table 3, the results of the 28 pasteurized milk samples and $10 \mathrm{PE}$ samples were analyzed using the CALUX bioassay and the HRGC/HRMS method. The differences between the results of CALUX $\times$ CFTEQ and WHO-TEQ (analyzed by HRGC/HRMS) were $0.012-0.748 \mathrm{pg}-\mathrm{TEQ} / \mathrm{g}$ fat, for an average of $0.246 \mathrm{pg}-\mathrm{TEQ} / \mathrm{g}$ fat. The CALUX $\times$ CF TEQ and the WHO-TEQ levels for pasteurized milk samples were statistically tested by the paired sample Student's $t$-tests with no statistically significant difference $(p=0.114)$. According to the statistical results, it was also performed that the CF value of 0.518 could be used to predict WHO-TEQ levels from HRGC/HRMS in pasteurized milk after the calculation of CALUX $\times$ CF TEQ.

Table 3 also shows that the PCDD/F WHO-TEQ levels in pasteurized milk samples are below the recommended EU noncommercialization threshold value of $3 \mathrm{pg}$-WHO-TEQ/g fat. The semi-quantitative results in 28 pasteurized milk samples and $10 \mathrm{PE}$ samples were examined in Table 3 . There is only one false positive sample (2.63\%) and no false negative samples. The false negative rate of the CALUX bioassay screening method is smaller than the criteria in the EU $(<1 \%)$ [25]. However, when CALUX bioassay is used as a screening method and the same false positive samples are in the confirmed area, the samples should be re-checked using HRGC/HRMS method. Future work is needed to validate that CALUX bioassay could be surveyed in the contaminated area especially for the hot spot areas. 
Table 3

The PCDD/F results for the pasteurized milk samples using the CALUX bioassay and the HRGC/HRMS method

\begin{tabular}{|c|c|c|c|}
\hline Sample number & XDS-CALUX (pg-TEQ/g fat) & $\mathrm{XDS}-\mathrm{CALUX} \times \mathrm{CF}^{\mathrm{a}}(\mathrm{pg}-\mathrm{TEQ} / \mathrm{g}$ fat $)$ & HRGC/HRMS (pg-WHO-TEQ/g fat) \\
\hline 1 & 2.927 & 1.197 & 0.743 \\
\hline 2 & 2.402 & 0.982 & 1.02 \\
\hline 3 & 3.399 & 1.390 & 1.11 \\
\hline 4 & 1.694 & 0.693 & 0.96 \\
\hline 5 & 2.318 & 0.948 & 0.983 \\
\hline 6 & 2.272 & 0.929 & 1.23 \\
\hline 7 & 1.671 & 0.683 & 0.55 \\
\hline 8 & 1.911 & 0.782 & 0.892 \\
\hline 9 & 0.996 & 0.407 & 0.918 \\
\hline 10 & 4.543 & 1.858 & 1.13 \\
\hline 11 & 2.008 & 0.821 & 0.912 \\
\hline 12 & 0.825 & 0.337 & 0.906 \\
\hline 13 & 1.759 & 0.719 & 0.936 \\
\hline 14 & 1.765 & 0.722 & 0.71 \\
\hline 15 & 1.469 & 0.601 & 0.55 \\
\hline 16 & 0.456 & 0.187 & 0.56 \\
\hline 17 & 0.878 & 0.359 & 0.189 \\
\hline 18 & 1.042 & 0.426 & 0.221 \\
\hline 19 & 1.251 & 0.512 & 0.532 \\
\hline 20 & 1.189 & 0.486 & 0.684 \\
\hline 21 & 1.227 & 0.502 & 0.878 \\
\hline 22 & 1.619 & 0.662 & 1.41 \\
\hline 23 & 0.87 & 0.356 & 0.55 \\
\hline 24 & 1.176 & 0.481 & 0.729 \\
\hline 25 & 2.324 & 0.951 & 1.01 \\
\hline 26 & 1.703 & 0.697 & 0.971 \\
\hline 27 & 1.368 & 0.560 & 0.7 \\
\hline 28 & 1.531 & 0.626 & 0.55 \\
\hline PE1-0 & 1.073 & 0.556 & 0.628 \\
\hline PE1-1 & 2.682 & 1.39 & 1.416 \\
\hline PE1-2 & 2.712 & 1.40 & 2.086 \\
\hline PE1-3 & 5.413 & 2.80 & 2.855 \\
\hline PE1-4 & 7.268 & 3.76 & 3.926 \\
\hline PE2-0 & 2.372 & 1.23 & 0.628 \\
\hline PE2-1 & 3.119 & 1.62 & 1.416 \\
\hline PE2-2 & 3.934 & 2.04 & 2.086 \\
\hline PE2-3 & 6.452 & 3.34 & 2.855 \\
\hline PE2-4 & 7.17 & 3.71 & 3.926 \\
\hline
\end{tabular}

${ }^{\text {a }} \mathrm{CF}=0.409$ for pasteurized milk samples; $\mathrm{CF}=0.518$ for $\mathrm{PE}$ samples.

\section{Conclusions}

The control chart for the QC chart and the STD6 standard (in the $\mu \pm 2 \sigma$ range), the LOD $(0.168 \mathrm{pg}-\mathrm{TEQ} / \mathrm{g}$ fat), and the LOQ $(0.335 \mathrm{pg}-\mathrm{TEQ} / \mathrm{g}$ fat $)$ were obtained from the dioxin standard solutions. The RSD (20.7\%), recovery efficiencies (averaged 79.9\%), Pearson's correlation coefficient (0.953), the accuracy $R$ (average 112\%), and the comparability RPD (average 19.0\%) of CALUX-TEQ were obtained from SM samples. The percentage of occurring false negative sample is $6.25 \%$, when using CALUX bioassay as a screening method for SM samples. Using CALUX bioassay as the screening method for pasteurized milk samples and PE samples, four samples $(10.5 \%)$ needed to be confirmed by the HRGC/HRMS method. CALUX-TEQs show no significant difference with dioxin-TEQs. The finding of this study proves that CALUX bioassay is a good screening tool and a potential semiquantitative method to determine the PCDD/F TEQ levels in pasteurized milk.

\section{Acknowledgements}

The authors would like to thank Mr. Jian-Yuan Yu, Mr. Fu-Guan Chen, and HIYOSHI Company (Japan) for technical support and the National Science Council in Taiwan for supporting this research work (Grant NSC 95-2221-E-230-013).

\section{References}

[1] Internal Agency for Research on Cancer, Polychlorinated dibenzo-paradioxins and polychlorinated dibenzofurans-summary of data reported and evaluation IARC Monogr. Eval. Carcinog. Risk Hum., vol. 69, IARC Press, Lyon, 1997.

[2] A. Schecter, L. Birnbaum, J.J. Ryan, J.D. Constable, Dioxins: an overview, Environ. Res. 101 (2006) 419-428.

[3] Clemson Extension's HG.IC, Food storage: refrigerator and freezer, Food Safety, Nutrition, Diet \& Health, 2004. http://hgic.clemson.edu/PDF/HGIC3522.pdf.

[4] R. Hoogenboom, T. Bovee, W. Traag, R. Hoogerbrugge, B. Baumann, L. Portier, G.v.d. Weg, J.d. Vries, The use of the DR CALUX bioassay and indicator polychlorinated biphenyls for screening of elevated levels of diox- 
ins and dioxin-like polychlorinated biphenyls in eel, Mol. Nutr. Food Res. 50 (2006) 945-957.

[5] R. Hoogenboom, The combined use of the CALUX bioassay and the HRGC/HRMS method for the detection of novel dioxin sources and new dioxin-like compounds, Environ. Sci. Pollut. Res. 9 (2002) 304-306.

[6] I. Windal, N.V. Wouwe, G. Eppe, C. Xhrouet, V. Debacker, W. Baeyens, E.D. Pauw, L. Goeyens, Validation and interpretation of CALUX as a tool for the estimation of dioxin-like activity in marine biological matrixes, Environ. Sci. Technol. 39 (2005) 1741-1748.

[7] T. Tsutsumi, Y. Amakura, M. Nakamura, D.J. Brown, G.C. Clark, K. Sasaki, M. Toyoda, T. Maitani, Validation of the CALUX bioassay for the screening of PCDD/Fs and dioxin-like PCBs in retail fish, The Analyst 128 (2003) 486-492.

[8] M.L. Scippo, G. Eppe, E. De Pauw, G. Maghuin-Rogister, DR-CALUX(R) screening of food samples: evaluation of the quantitative approach to measure dioxin, furans and dioxin-like PCBs, Talanta 63 (2004) 1193-1202.

[9] H. Vanderperren, N. Van Wouwe, S. Behets, I. Windal, I. Van Overmeire, A. Fontaine, TEQ-value determinations of animal feed; emphasis on the CALUX bioassay validation, Talanta 63 (2004) 1277-1280.

[10] M. Warner, B. Eskenazi, D.G. Patterson Jr., G. Clark, W.E. Turner, L. Bonsignore, P. Mocarelli, P.M. Gerthoux, Dioxin-like TEQ of women from the Seveso, Italy area by ID-HRGC/HRMS and CALUX, J. Expos. Anal. Environ. Epidemiol. 15 (2005) 310-318.

[11] N.V. Wouwe, I. Windal, H. Vanderperren, G. Eppe, C. Xhrouet, A.-C. Massart, N. Debacker, A. Sasse, W. Baeyens, E.D. Pauw, F. Sartor, H.V. Oyen, L. Goeyens, Validation of the CALUX bioassay for PCDD/F analyses in human blood plasma and comparison with GC-HRMS, Talanta 63 (2004) 1157-1167.

[12] P. Laier, T. Cederberg, J.C. Larsen, A.M. Vinggaard, Applicability of the CALUX bioassay for screening of dioxin levels in human milk samples, Food Addict. Contamin. 20 (2003) 583-595.

[13] U.S. EPA, Technologies for monitoring and measurement of dioxin and dioxin-like compounds in soil and sediment: CALUX ${ }^{\circledR}$ by XDS, Innovative Technology Verification Report, EPA/540/R-05/001, 2005.

[14] H.R. Chao, T.C. Tsou, L.A. Li, F.Y. Tsai, Y.F. Wang, C.H. Tsai, E.E. Chang, Z.F. Miao, C.H. Wu, W.J. Lee, Arsenic inhibits induction of cytochrome P450 $1 \mathrm{~A} 1$ by $2,3,7,8$-tetrachlorodibenzo- $p$-dioxin in human hepatoma cells, J. Hazard. Mater. 137 (2006) 716-722.
[15] H.R. Chao, Y.F. Wang, H.T. Chen, Y.C. Ko, E.E. Chang, Y.J. Huang, F.Y Tsai, C.H. Tsai, C.H. Wu, T.C. Tsou, Differential effect of arecoline on the endogenous dioxin-responsive cytochrome P450 1A1 and on a stably transfected dioxin-responsive element-driven reporter in human hepatoma cells, J. Hazard. Mater. 149 (2007) 234-237.

[16] M. Asari, H. Takatsuki, M. Yamazaki, T. Azuma, H. Takigami, S.I Sakai, Waste wood recycling as animal bedding and development of biomonitoring tool using the CALUX assay, Environ. Int. 30 (2004) 639649.

[17] Japan Ministry of the Environment, Bioassay method manual for dioxin-like compounds (Japanese), http://www.env.go.jp/chemi/dioxin/ guide/sim_method/full.pdf, 2006.

[18] Taiwan EPA, Analysis method of dioxins and furans: isotope dilution high resolution gas chromatography-mass spectrometry (Chinese), NIEA M801.11B, 2005 .

[19] U.S. EPA, Tetra- through octa-chlorinated dioxins and furans by isotope dilution HRGC/HRMS, Revision B, Method 1613, 1994.

[20] D.J. Brown, L. Goeyens, I.V. Overmeire, M. Chu, H. Murata, G.C. Clark, Quality control criteria implemented for monitoring the use of the CALUX bioassay, Organohalogen Compd. 54 (2001) 32-35.

[21] D.C. Montgomery, Introduction to Statistical Quality Control, fourth ed., John Wiley, New York, NY, 2001.

[22] L. Hoogenboom, W. Traag, T. Bovee, L. Goeyens, S. Carbonnelle, J. van Loco, H. Beernaert, G. Jacobs, G. Schoeters, W. Baeyens, The CALUX bioassay: current status of its application to screening food and feed, Trends Anal. Chem. 25 (2006) 410-420.

[23] H. Besselink, A. Jones, M. Pijnappels, A. Swinkels, K. Fjellanger, A. Brouwer, Comparison of DR CALUX and HRGCMS-derived TEQ's: introduction of conversion factors, Organohalogen Compd. 60 (2003) 203206.

[24] I.V. Overmeire, J.V. Loco, P. Roos, S. Carbonnelle, L. Goeyens, Interpretation of CALUX results in view of the EU maximal TEQ level in milk, Talanta 63 (2004) 1241-1247.

[25] European Commission, Directive 2002/69/EC of 26 July 2002 laying down the sampling methods and the methods of analysis for the official control of dioxins and the determination of dioxin-like PCBs in foodstuffs, Official Journal of the European Communities, L 209/5, 2002. 